\title{
Anabases
}

ANABASES Traditions et réceptions de l'Antiquité

$16 \mid 2012$

Varia

\section{La date et le sens du voyage de Rutilius Namatianus}

Jérôme Carcopino

\section{OpenEdition}

Journals

Édition électronique

URL : http://journals.openedition.org/anabases/3987

DOI : 10.4000/anabases.3987

ISSN : 2256-9421

\section{Éditeur}

E.R.A.S.M.E.

\section{Édition imprimée}

Date de publication : 1 octobre 2012

Pagination : 241-263

ISSN : 1774-4296

\section{Référence électronique}

Jérôme Carcopino, "La date et le sens du voyage de Rutilius Namatianus », Anabases [En ligne], 16 |

2012, mis en ligne le 01 octobre 2015, consulté le 20 octobre 2019. URL : http://

journals.openedition.org/anabases/3987 ; DOI : 10.4000/anabases.3987 
Anabases 16 (2012), p. 241-263.

\section{La date et le sens du voyage de Rutilius Namatianus*}

Jérôme CARCOPINO

Pour le commun des LetTrés, Rutilius Namatianus est le poète gaulois, probablement originaire de Toulouse ${ }^{1}$, auquel nous devons le plus bel hommage qui ait été rendu à la grandeur romaine, dans le temps même où elle allait sombrer dans la tempête des invasions barbares. Qui ne se souvient, en effet, de sa superbe prosopopée ? "O Rome, tu as donné une unique patrie à des peuples divers. Tu as été la bienfaitrice des méchants que conquit ta domination. En offrant à tes vaincus le partage de tes droits, tu n'as fait qu'une ville de ce qui était le monde entier :

Fecisti patriam diversis gentibus unam;

Profuit iniustis te dominante capi.

Dumque offers victis propria consortia iuris,

Urbem fecisti quod prius orbis erat ${ }^{2}$."

Comme l'a écrit René Pichon, "ce sont peut-être les plus beaux vers que Rome ait jamais inspirés, ceux qui marquent le mieux sa double mission conquérante et civilisatrice ${ }^{3}$ ». En vérité, en ces vers d'un sincère enthousiasme, s'élève le chant du cygne de la latinité.

"La date et le sens du voyage de Rutilius Namatianus ", in Rencontres de l'histoire et de la littérature romaines, Paris, 1963, p. 233-270 (parution initiale : "Chronologie et histoire littéraire ", Revue des Études latines 6 (1928), p. 180-200.

1 Cf. PréCHAC, Rutilius Namatianus, Sur son retour, Paris, 1933, p. vi. De fait, Toulouse est nommée dans le poème I, 596 ; cf. Vesseheau, Rutilius Namatianus, Paris, 1904, p. 153 et suiv. Cf. infra, p. 258, n. 82 [ici : p. 255, n. 82].

2 I, 63-66.

3 PichON, Histoire de la littérature latine, p. 825. 
En revanche, pour l'érudition contemporaine, le poème où ils s'inscrivent, cousus sur la trame d'un récit de voyage, soulève de multiples difficultés d'interprétation.

D'abord, sujet et manière, il rappelle, soit la Satire où Horace nous a malicieusement décrit les étapes qui l'ont acheminé, en compagnie de Mécène, depuis Rome jusqu'à Brindes ${ }^{4}$, soit encore l'élégie où Ovide, sur le bateau secoué par les houles de décembre, nous a plaintivement conté son orageuse traversée de l'Adriatique ${ }^{5}$; et " il ressemble à un journal versifié en cours de route ${ }^{6}$ ". Mais les digressions dont il abonde, les morceaux de bravoure, louanges ou invectives, qu'il renferme et dont on a du mal à penser qu'ils n'aient pas été composés à tête reposée ont tôt fait d'effacer cette ressemblance ${ }^{7}$. Comment nous faudra-t-il choisir entre ces apparences contradictoires?

En outre, nous ne sommes pas capables de désigner nommément avec certitude l'œuvre que Rutilius nous a laissée. Sur le plus ancien de nos manuscrits, le Vindobonensis, exécuté avant 1501, elle est identifiée avec ce qui reste d'un poème consacré par Rutilius à son retour en Gaule : de reditu suo in Galliam Narbonen(sem) ${ }^{8}$. Le manuscrit Romanus, de quelque trente ans postérieur, nous apprend, au contraire, que, dédié à Caeionius Rufius Volusianus, le poème portait en tête de son livre premier le titre d'Itinerarium. En ce qui concerne la dédicace, il est permis d'hésiter : elle n'est peut-être que la glose tirée par un copiste érudit du passage où Rutilius promet à son ami de le faire cheminer, porté par son vers, non point sous le nom de Caeionus, réfractaire à la prosodie, mais sous le surnom de Rufius, sous lequel il l'a déjà chanté sur une autre page :

Cognomen versu veheris, carissime Rufi,

Illo te dudum pagina nostra canit? .

Mais peut-être aussi bien la dédicace, à laquelle ce distique pourrait faire allusion, figurait-elle réellement sur le texte original.

Quant au titre, que cite le copiste du Romanus, il est difficile de l'accepter tel quel. Le substantif Itinerarium ne saurait se suffire à lui-même et appelle un qualificatif que le Romanus a omis ou estropié, mais que Vollmer a restitué sous la forme Iter Gallicum $^{10}$ : et nous nous demandons comment sortir de l'impasse où nous enfoncent les dissonances de la tradition manuscrite.

4 Hor., Sat., I, 5.

5 Ov., TV., I, 13, 3-4 ; cf. ibid., I, 10 et ajouter Stace, Silves, III, 2.

6 PRÉCHAC, op. cit., p. XIX.

7 La louange de Rome, par exemple, ou l'invective contre Stilicho, II, 41 et suiv.

8 Cf. l'apparat critique ap. PrÉCHAC, op. cit., p. 2.

9 I, 421-422. Ce personnage est cité, en effet, dans un passage antérieur : I, 167-170. Mais si pagina désigne ici le premier feuillet, le scribe du Romanus aurait eu raison, cf. infra, p. 269 [ici : p. 262].

10 Vollmer, P.W., I ${ }^{\text {A }}$ c. 1252. 
Enfin et surtout, il y a un déséquilibre choquant, inadmissible, entre les deux livres dont se compose le poème : au premier, qui comprend 644 vers, ne fait pas contrepoids le second, qui n'en compte que... 68 ! Sommes-nous en présence d'un texte inachevé, comme le pensait Vessereau ${ }^{11}$, ou d'un texte que son auteur avait conduit à son terme mais qui, suivant Pierre de Labriolle, "se dérobe à nous dans le moment où le poète s'apprêtait à prendre un nouvel élan ${ }^{12}$ ", parce qu'il a été mutilé par la paresse de ses scribes ou la perte de ses derniers feuillets.

Ce sont là autant de questions où s'affrontent et s'entrechoquent arguments et conjectures et que j'espère pouvoir résoudre grâce à l'étude où j'ai établi, jour après jour, la chronologie du voyage de Rutilius ${ }^{13}$.

Depuis lors, mes conclusions ont été admises et M. François Préchac les a incorporées dans ses compléments à l'édition, préparée par feu Vessereau, du poème de Rutilius dans la collection Guillaume Budé ${ }^{14}$; et il a même cru devoir, en une note spécialement rédigée à leur appui, légitimer mon « intervention ", qui, " même après Tillemont, était nécessaire ${ }^{15}$ \%. Aussi m'excusera-t-on de reprendre aujourd'hui mes conclusions d'alors, pour les fortifier et en déduire, mieux que je ne l'avais fait, les conséquences, que, par la solution des problèmes posés, elles entraînent dans la conception que nous devrons former désormais des intentions de Rutilius Namatianus et de la façon dont le poète les a réalisées.

\section{I}

Toute fausse modestie mise à part, je suis en effet, forcé de constater qu'antérieurement à mon travail et à celui de M. François Préchac, qui l'a utilisé, la chronologie régnante, celle que, d'après Scaliger, Zumpt avait établie ${ }^{16}$, il y a plus d'un siècle,

11 Vessereau, op. cit., p. 254.

12 De Labriolle, R.E.L., 1928, p. 31.

13 Cf. J. Carcopino, Chronologie et histoire littéraire. À propos du poème de Rutilius Namatianus, dans la R.E.L., 1928, p. 180-200.

14 PrÉCHAC, op. cit., p. XV-XIX.

15 Ibid., p. XIII, n. 5 : «Carcopino de qui l'intervention, même après Tillemont, était nécessaire, etc. " D'autant plus nécessaire, oserai-je ajouter, qu'il suffit de citer Tillemont pour savoir combien ses intuitions manquaient à la fois d'exactitude et de justifications. Cf. par ex., Tillemont, Histoire des Empereurs, Bruxelles, 1710, p. 277 : "Ce fut, apparemment, à la fin de l'an 417 ou de 420 que Rutilius s'en retourna vers les Gaules » et ibid., p. 287 : « Il (Rutilius) partit comme nous l'avons dit ci-devant ou en 417, ou en 420, vers le mois d'octobre... » Tillemont s'est uniquement fondé sur le fait qu'en 416 et en 418 la praefectura Urbi ont [sic: a] d'autres titulaires ; cf. infra, p. 243.

16 Zumpt, Observationes in Rutilii Claudii Namatiani carmen de reditu suo, Berlin, 1937, ch. III. 
et qu'ensuite lui empruntèrent Vessereau, chez nous ${ }^{17}$, Vollmer en Allemagne ${ }^{18}$, Ussani en Italie ${ }^{19}$, ne repose que sur des erreurs. Erreur, sur l'année, pour commencer. Ussani, Vollmer, Vessereau s'accordent pour assigner à l'année 416 le voyage de Rutilius. Le poète n'a-t-il pas, en effet, pris soin de nous confier qu'au moment où il la quittait avec un poignant regret, Rome avait atteint 1169 ans ? " Puisses-tu, seule, ô Rome, ne pas craindre pour le fil de ton destin, encore qu'à onze cent soixante ans accomplis tu en ajoutes neuf autres encore!

Solaque fatales non vereare colos,

Quamvis sedecies denis et mille peractis

Annus praeterea iam tibi nonus erat ${ }^{20}$."

L'ère de la fondation de Rome commence en une année qui correspond à 754 avant notre ère. D'où il suit que par la soustraction conséquente de 753 ans de notre ère à ce nombre de 1169 années de Rome, Rutilius a regagné sa Gaule natale en 416 ap. J.-C. Le raisonnement semble irréfutable et la conclusion péremptoire ; et, pourtant, il m'est impossible d'y souscrire.

C'est que la transition de l'ère de Rome à notre ère chrétienne ne s'opère pas avec cette simplicité automatique. Selon les calculs de l'érudition antique, la fondation de Rome était généralement attribuée à la troisième année de la $\sigma^{\mathrm{e}}$ olympiade. Mais le début de l'année civile romaine, au $1^{\mathrm{er}}$ janvier, précédait de huit mois celui de l'année Olympique, communément fixée, sous l'Empire, au $1^{\text {er }}$ septembre. L'année 3 de la $6^{\text {e }}$ olympiade chevauchait donc sur deux années civiles romaines; et, rapportée à l'ère chrétienne, elle ne pouvait se définir exactement, ni par l'année 754 av. J.-C., ni par l'année 753 av. J.-C. Pratiquement, et pour simplifier leurs calculs, les Romains choisissaient entre les composantes de cette année mixte. Varron opta pour la plus ancienne des deux, d'où l'ère Varronienne, qui débute en 754 av. J.-C. Mais parallèlement, et peut-être pour rendre hommage à la tradition qui commémorait au 21 avril le jour de la fondation de la Ville Éternelle, le rédacteur des Fastes consulaires et triomphaux gravés, par ordre d'Auguste, sur les murs de la Regia, a choisi la plus récente ; d'où l'ère des Fastes, qui débute en 753 av. J.-C. Pendant toute la durée de l'Empire, les computistes ont oscillé d'un système à l'autre, et leurs deux méthodes n'ont cessé d'être concurremment suivies, pour ainsi dire, au petit bonheur, non seulement par les historiens, mais dans les pièces officielles. Les exemples abondent de ce double emploi, ainsi que l'a démontré Kubitscheck ${ }^{21}$. Je me bornerai à reproduire ici les plus significatifs.

17 Vessereau, op. cit., p. 253 et suiv.

18 Vollmer, s. v ${ }^{\circ}$, Rutilius, P.W., I- ${ }^{\mathrm{A}}$, c. 1251.

19 Ussani, Roma e Gallia, dans Atene e Roma, 1916, p. 213.

20 I, 134-136.

21 KubitscheK, s. vº Aéra, P.W., I, c. 622. 
Tacite, dans les Annales, place sous le quatrième consulat de Claude, soit en 47 de notre ère, la célébration, par cet empereur, des ludi saeculares. Or, il nous donne lui-même cette précision que ces jeux ont été donnés huit cents ans après la fondation de Rome : (anno) octingentesimo post Romam conditam ${ }^{22}$. En ce passage de ses Annales, Tacite a emprunté l'ère varronienne ${ }^{23}$. Ouvrons maintenant les Histoires du même Tacite. Dès la première phrase ${ }^{24}$, l'auteur nous avertit qu'il commence son ouvrage au deuxième consulat de Galba, soit au $1^{\text {er }}$ janvier 69 ap. J.-C. et il ajoute qu'à cette date 820 années s'étaient écoulées depuis la fondation de Rome. Le $1^{\text {er }}$ janvier 69 ap. J.-C., inaugure donc ici la $821^{\mathrm{e}}$ année Urbis conditae; et infidèle, cette fois, au système varronien, Tacite, dans ses Histoires, s'est approprié l'ère des Fastes.

Passons aux pièces d'archives. Même usage alternatif, arbitraire, des deux chronologies rivales. À Rome, dans les Procès verbaux des Frères Arvales, les dates correspondant à $68,71,153,210,217,219^{25}, 255^{26}$ ap. J.-C, sont supputées d'après l'ère varronienne ; et, en revanche, les années, correspondant aux années 92, 115, 161, 19727, $198,200^{28}, 202^{29}, 218,221^{30}$ av. J.-C., le sont d'après l'ère des Fastes. Pareillement, sur un même fragment de la chronique municipale de Bovillae (Le Fratocchie, sur la voie Appienne, à l'Ouest du Lac d'Albano), les consulats de l'an 200 ap. J.-C., sont rapportés à l'an 953 de Rome, soit à l'ère varronienne et ceux de l'an 180 ap. J.-C., à l'an 932 de Rome, soit à l'ère des Fastes ${ }^{31}$.

Dans ces conditions, il nous est interdit d'affirmer que l'an 1169 de Rome, où Rutilius entreprit son voyage de retour en Gaule, doive être nécessairement converti en l'an 416 de notre ère. C'est l'an 416, si Rutilius a fondé ses calculs sur l'ère varronienne. C'est l'an 417, si, au contraire, ils reposent sur l'ère des Fastes. Par conséquent, a priori, le millésime de 1169 Urbis conditae, unique dans son poème, se rattache indifféremment à l'un ou à l'autre des deux computs, et entre les deux transpositions qu'ils autorisent nous n'aurions aucune raison de décider, si, par bonheur, le contexte ne nous contraignait point à éliminer 416 au bénéfice de 417 .

22 TAC., Ann., XI, 11.

23 De même, dans la Germania, XXXVII, la $640^{\mathrm{e}}$ année Urbis conditae correspond au consulat de Caecilius Metellus et de Papirius Carbo, qui date de 113 av. J.-C. Tacite, dans ce passage, s'est donc référé à l'ère varronienne.

24 TAC., Hist., I, 1.

25 C.I.L., VI, 1984.

26 Ibid., 2001.

27 Ibid., 1984.

28 Ibid., 2004.

29 Ibid., 1984.

30 Ibid., 2001.

31 C.I.L., XIV, 2393. 
Plus loin, en effet, Rutilius raconte qu'au cinquième jour de sa traversée, il a appris la nomination de son jeune ami Rufius ${ }^{32}$ à cette préfecture de la Ville, dont lui-même, pendant quelques mois de l'année 414 , avait été l'heureux titulaire ${ }^{33}$ : «Ici (à Populonia), une joyeuse nouvelle parvient jusqu'à nos oreilles; et peu s'en fallut alors que ma résolution fût prise de m'en retourner à Rome. Ici, en effet, nous sommes informés que la préfecture de la Ville sainte vient d'être confiée à tes mérites, ô mon doux ami ! Je souhaiterais enchâsser ton nom propre dans mon poème. Mais il est certains pieds que repousse une règle sévère. Sous ton surnom du moins auras-tu paru dans mes vers, ô mon cher Rufius, et c'est avec lui que je t'ai déjà chanté sur une autre page :

Laetior hic nostras crebrescit fama per aures:

Hic praefecturam sacrae cognoscimus Urbis

Delatam meritis, dulcis amice, tuis.

Optarem verum complecti carmine nomen.

Sed quosdam refugit regula dura pedes.

Cognomen versu veheris, carissime Rufi;

Illo te dudum pagina nostra canit ${ }^{34}$."

Un peu plus haut, en effet, Rutilius s'était attendri au souvenir de cette prédilection :

"Mes amis m'accompagnent. Bientôt les autres rentrent à Rome; mais, à mes pas s'attache Rufius, gloire vivante de son père Albinus ${ }^{35}$, Rufius dont le nom remonte à l'antique Volusus ${ }^{36}$ et en qui revivent les rois rutules désignés par Virgile. Son éloquence lui a valu le commandement du Palais. Oui, à la fleur de l'âge, il a été jugé digne de parler au nom de l'Empereur ; et auparavant il avait comme enfant, gouverné les Carthaginois en qualité de proconsul :

.. Comitantur amici ;

Iamque aliis Roman redeuntibus haeret eunti

32 PréCHAC, op. cit., l'appelle Rufius Antonius Agrypius Volusianus. Le personnage portait, en effet, tous ces surnoms, mais son gentilice, réfractaire à la métrique (cf. le texte suivant) était Caeionius ; cf. SEeck, P.W., IV, c. 1866 et PréChac, op. cit., p. IX, n. 1.

33 La préfecture de Rutilius, qui a eu Caecina Albinus pour successeur (I, 467), se place avant le 17 septembre 414, date à laquelle une constitution d'Honorius est adressée à ce dernier (Cod. Theod., XIII, 5, 38), et après le 12 janvier 414, date à laquelle Eutychianus en était encore le titulaire (Cod. Theod., Fil, 8, 111). Tout cela a été parfaitement débrouillé par Vessereau, op. cit., p. 179 et par Chastagnol, Les fastes de la préfecture de Rome au Bas-Empire, Paris, 1962, p. 273-275.

34 I, 415-422. Sur le dernier vers et le sens de pagina, cf. supra, p. 235 [ici : p. 242]. Au vers précédent, j'ai adopté l'élégante correction de Préchac : veheris, qui évite la faute de quantité de veneris.

35 Sur le personnage, cf. Préchac, op. cit., p. IX et SEeCK, P.W., IV, c. 1864.

36 À cause de son surnom de Volusianus, cf. supra, p. 235 [ici : p. 242]. Volusus est un chef volsque allié de Turnus dans VIrg., Aen., XI, 463. 


\author{
Rufius, Albini gloria viva patris, \\ Qui Volusi antiquo derivat stemmate nomen, \\ Et reges Rutulos teste Marone refert. \\ Huius facundae commissa palatia linguae. \\ Primaevus meruit principis ore loqui. \\ Rexerat ante puer populos pro consule Poenos ${ }^{37}$."
}

Rétablissons en prose ce que furent la carrière et l'identité du personnage. Tout jeune, il a été proconsul d'Afrique, en résidence à Carthage. Puis il a été appelé à la questure du Palais, dont les titulaires traduisent en leurs discours la volonté du Prince. Son nom propre - son gentilice - Caeionius, rebelle à la métrique, nous échappe dans les deux passages qui le concernent. Mais à son surnom de Rufius, qu'ils énoncent l'un et l'autre, celui-ci ajoute un surnom qui tiré de Volusus, ne saurait être que Volusianus, et un des surnoms que portait déjà son père : Albinus. Il n'en faut pas davantage pour le reconnaître en toute certitude. Il s'agit du fils de Caeionius Albinus, qui, préfet de la Ville en 389-391 38 , était désigné par toute une kyrielle de cognomina: Rufius Antonius Agrypius Volusianus. Lui-même, préfet du prétoire d'Italie en 428-429 ${ }^{39}$, avait été investi deux fois auparavant de la préfecture de la Ville : la deuxième fois, en $421^{40}$; la première fois, lors du voyage de Rutilius ; et c'est précisément cette coïncidence qui, des deux années où ce voyage est possible, va exclure l'une et imposer l'autre.

En effet, comme l'avait déjà indiqué Tillemont ${ }^{41}$, Rufius Volusianus n'a pu être nommé préfet de la Ville dans l'automne ou l'hiver de 416, parce qu'une constitution impériale du Code Théodosien fut adressée le 14 récembre 416 au préfet de la Ville qui était alors Probianus ${ }^{42}$. En vain supposerait-on, avec Vessereau ${ }^{43}$, que Rufius Volusianus, entré en charge en 417 , avait été désigné plusieurs mois à l'avance pour succéder à Probianus. La préfecture de la Ville n'est pas une magistrature à laquelle on est destiné par anticipation. C'est une fonction que l'on exerce aussitôt qu'on en est revêtu par la faveur du Prince ; et Rutilius a failli retourner à Rome pour avoir le bonheur d'y vivre sous l'autorité de son meilleur ami. La nouvelle de cette promotion est donnée au passé - delata ${ }^{44}$. Quand Rutilius en est informé, Rufius est déjà en possession de cet honneur - honor - et du pouvoir - potestas ${ }^{45}$ - qu'il implique ; et il semble tout de suite à Rutilius, qui les avait détenus avant Rufius, qu'il est en train

37 I, 165-173.

38 Cf. Seeck, P.W., c. 1864, n 33 et Chastagnol, op. cit., p. 233-236.

39 Seeck, P.W., IV, c. 1866. Cf. Chastagnol, op. cit., p. 277-279.

40 Haenel, Corpus Legum, p. 240. Cf. C.I.L., VI, 1194 et 1661.

41 Tillemont, Histoire des Empereurs, V, note XuIII, p. 84.

42 Cod. Theod., XIV, 2, 4, 4.

43 Vessereau, op. cit., p. 256.

44 I, 418.

45 I, 426-428. 
de les partager avec lui, ou plutôt de les assumer à nouveau en la personne de cet autre lui-même : "On a promu une grande partie de mon âme. Voilà, oui voilà comment je dois me plaire à voir renouveler ma puissance par celui que j'avais souhaité et dont la promotion redouble le bonheur de la mienne :

Provecta est animae portio magna meae

Sic mihi, sic potius placent geminata potestas,

Per quem malueram rursus honore fruor ${ }^{46}$."

Concluons : en 416, la préfecture de la Ville n'était pas vacante. La place était prise par Probianus. Après Probianus, au contraire, et avant Symmaque qui y est installé en $418^{47}$, elle est libre. C'est donc en 417 que Rufius Volusianus y a été nommé, et en 417, par conséquent, soit en l'an 1169 de Rome, calculé d'après l'ère officielle des Fastes, que Rutilius s'en est allé rejoindre la Gaule, sa patrie.

Aussi bien, cette date cadre-t-elle avec les intentions et les sentiments du poète. Celui-ci rentrait dans son pays natal avec le généreux propos d'y relever les ruines amoncelées par les guerres : "Oui, il est temps quand nos terres ont été ravagées par de cruels incendies, de rebâtir, ne fût-ce que des cabanes de bergers :

Iam tempus laceris saeva incendia fundis Vel pastorales aedificare casas ${ }^{48}$."

Cet effort de reconstruction exigeait que les hostilités eussent pris fin, que l'ordre et la paix eussent été ramenés en Gaule. Or, après les années 408 et suivantes, années de déprédations, de ruines et d'épouvante, où il semblait aux Gaulois qu'approchait la fin du monde ${ }^{49}$, la Gaule n'a commencé de respirer qu'en 417, lorsque le général d’Honorius, qui avait poursuivi la restauration du pays, Constance, le futur Constance III, a reçu, pour récompense de ses services, probablement dans Arles, où il avait installé son quartier général, le titre de patrice et la main de Placidie qu'il venait de tirer de captivité. En juin 416, il s'est emparé de l'usurpateur Attale ; à la fin de 416, il se débarrasse du roi vandale Fredbal ; et, en 418, l'armistice qu'il avait conclu avec Wallia, roi des Goths, sera transformé par ses soins en la paix définitive grâce à laquelle, de nouveau, se réunira, dans Arles, l'assemblée, ou concilium, des sept provinces gauloises ${ }^{50}$.

46 Ibid.

47 Cf. Tillemont, op. cit., loc. cit. ; Consini, De praefectis Urbis, Pise, 1766, p. 366-368 ; Chastagnol, op. cit., p. 279-281.

48 I, 29-30.

49 Cf. les textes invoqués dans le beau livre de Christian Courtois, Les Vandales et l'Afrique, Paris, 1955, p. 50, n. 1 et 2.

50 Cf., sur Constance III, mon mémoire Choses et gens du pays d'Arles, dans la Revue du Lyonnais, 1922. 
Rutilius se reproche, en ses vers, d'avoir trop tardé à venir au secours de sa patrie pacifiée. En 416, alors que brûlaient encore maints foyers de révolte et de guerre et que l'avenir demeurait incertain, ses scrupules auraient été prématurés. À la fin de 417 , ils se comprennent de soi, sans cesser de témoigner de sa délicatesse et de son patriotisme ${ }^{51}$.

Je dis : à la fin de 417, car, ainsi que nous l'allons voir, il est possible de reconstituer, à vingt-quatre heures près, l'emploi du temps de Rutilius, depuis son départ de Rome et son embarquement au Portus Ostiensis, jusqu'à son débarquement à Luna. Mais il convient, pour cela, de renoncer aux méthodes de calcul, à la fois trop brutales et trop superficiellement informées, qui ont, jusqu’à présent, faussé le cadre de ses pérégrinations. Rutilius, pourtant, les a déroulées avec une précision suffisante pour que nul ne puisse se méprendre sur la chronologie relative de son voyage. Il s'est embarqué, dans le Port d'Ostie, à l'aube du seizième jour qui suivit son départ de Rome : «Durant trois fois cinq jours, nous guettâmes le moment d'avoir confiance en la mer, jusqu'à ce que, rassurée par la nouvelle lune, se levât une brise meilleure :

Explorata fides pelagi ter quinque diebus,

Dum melior lunae fideret aura novae ${ }^{52}$."

Après quoi, il ajoute : "Nous appareillons à la lumière indécise de l'aurore, quand, rendue aux campagnes, la couleur commence de les faire reconnaître :

\section{Solvimus aurorae dubio quo tempore primum}

Agnosci patitur redditus arva color ${ }^{5}$."

Dès lors, jusqu'à Vada tout au moins, son retour s'est effectué en autant d'escales que de jours. Chaque matin, dès l'aube, il lève l'ancre ; puis, dans le courant de la journée, en des heures dont les chiffres varient avec, le vent et la distance des plus prochains mouillages, mais toujours avant la tombée de la nuit, il s'arrête, passe à terre la fin de l'après-midi tout entière et la nuit suivante ; puis, il recommence le lendemain. Le premier jour, il a ainsi vogué du Portus Ostiensis (Porto) à Centumcellae (Cività Vecchia) ; le deuxième de Centumcellae à Portus Herculis (Porto Ercole) ; le troisième, de Portus Herculis à l'embouchure de l'Umbro (Ombrone); le quatrième, de l'Umbro à Faleria (Porto di Falesia). Le cinquième jour, il s'est arrêté à Populonia. Le sixième, il a abordé à Vada, où il fut retenu, probablement un jour, par l'état de la mer. De là,

51 À cette date, le Toulousain Victorinus et le Trévire Protadius sont encore en Italie ; cf. infra, p. 258-259 [ici : p. 255-256].

52 I, 205-206.

53 I, 217-218. 
il gagne le port de Pise, où la tempête le force à séjourner quelque temps, avant de se rembarquer pour Luna, où se termine son poème, du moins tel qu'il est arrivé jusqu'à nous. Laissons provisoirement de côté ces trois dernières escales, puisque le poète, en négligeant de leur assigner une place nettement déterminée par rapport aux précédentes, ouvre ici quelque marge à la conjecture. Ne considérons que la section qu'il a jalonnée d'indiscutables repères. Elle s'étend sur sept étapes, dont la première, au Portus Ostiennis [sic: Ostiensis], a absorbé quinze jours pleins - ter quinque diebus ${ }^{54}$ - mais dont les six autres - Centumcellae, Portus Herculis, Umbro, Faleria, Populonia, Vadase répartissent, à raison d'une escale par jour, sur les six jours suivants. Il est clair, par conséquent, que si l'on pouvait fixer l'une d'entre elles, on rétablirait du même coup la chronologie absolue de toutes les autres; et c'est bien à quoi s'est ingéniée la critique moderne. Seulement, elle a débuté, dans cette voix excellente, par un faux-pas.

Depuis Scaliger ${ }^{55}$, en effet, on admet que Rutilius a quitté Rome à l'équinoxe d'automne : "Déjà Phoebus avait allongé la durée des heures nocturnes :

\section{Et iam nocturnis spatium. laxaverat horis $\left[\right.$ Phoebus ${ }^{56}$...»}

Les anciens fixaient au 24 septembre l'équinoxe d'automne. En conséquence, Scaliger, Zumpt ${ }^{57}$ et leurs épigones, sont d'avis que Rutilius, ayant laissé Rome le 24 septembre, s'est embarqué au Portus Ostiensis le 9, ou mieux le 10 octobre. D'aucuns, pour échapper aux difficultés que soulève ce comput et que nous signalerons tout à l'heure, prêtent à Rutilius leurs connaissances astronomiques et avancent le départ du voyageur de deux ou trois jours, comme l'équinoxe même. "Il est probable, affirme Vessereau, que Rutilius quitta Rome le 21 ou 22 septembre, ce qui reporte son embarquement au 6 ou 7 octobre $^{58}$ ", et Vollmer a repris textuellement la même opinion ${ }^{59}$. Or, il n'est que de se reporter aux vers précités, fondement vacillant et exigu de toute cette construction, pour s'apercevoir qu'ils ne sauraient la supporter. Le poète n'y a point dit être sorti de Rome le jour de l'équinoxe. Il rappelle, au contraire, qu'il s'est mis en route, alors que, déjà, Phoebus avait laissé du champ aux heures nocturnes. À l'équinoxe, comme eût dit M. de la Palisse, les nuits s'équilibrent avec les jours. Quand Rutilius a pris congé de la Ville Eternelle, les nuits étaient devenues plus longues que les jours; et le plus-que-parfait dont a usé le poète - laxaverat- marque avec force que cet allongement des nuits était accompli depuis quelque temps déjà. Ce n'est donc, évidemment, ni le 21 , ni le 22 , ni le 24 septembre, mais quelques semaines après,

54 I, 205 ; cf. supra, p. 246 [ici : p. 249].

55 SCAliger, Prooemium ad Eusebii chronica, cité par Vessereau, op. cit., p. 256, n. 2.

56 I, 183-184.

57 ZuMPT, op. cit., ch. III.

58 Vessereau, op. cit., p. 257.

59 Vollmer, P.W., I ${ }^{\text {A }}$, c. 1251 : «Rutilius wird am einundzwanzigsten oder am zweiundzwanzigsten september Rom verlassen haben. » 
alors que le raccourcissement des jours était déjà devenu sensible. Les vers auxquels les érudits ont voulu attacher une date déterminée, sans d'ailleurs parvenir à s'entendre sur elle, ne renferment, à les interpréter correctement, qu'un terminus a quo, un point de départ assez vague et flottant lui-même ! Force nous est de chercher mieux.

L'indication ferme que l'équinoxe d'automne nous refuse, je l'ai demandée, pour ma part, au spectacle qui a frappé Rutilius, à Faleria, au quatrième jour de sa navigation, au dix-neuvième jour de son départ de Rome. "Alors, aux carrefours de cette rustique bourgade, les paysans hilares se délassaient de leurs fatigues par des réjouissances sacrées : c'est ce jour-là qu'Osiris, enfin ressuscité, fait lever la féconde semence pour des moissons nouvelles :

Et tum forte hilares per compila rustica pagi

Mulcebant sacris pectora fessa iocis :

Illo quippe die tandem revocatus Osiris

Excitat in fruges germina laeta novas ${ }^{60}$."

Qu'on parvienne seulement à situer à sa place sur le calendrier religieux de l'Empire romain cette fête des semailles du culte osirien et le problème sera résolu.

Or il n'est pas trop malaisé de l'identifier sans chance d'erreur et de la dater en toute sécurité. Sur le temps normal des semailles dans l'Italie ancienne, point de doute : c'est le mois de novembre. Là-dessus les préceptes des agronomes concordent avec les éphémérides populaires que l'épigraphie nous a conservées. On lit sur l'inscription romaine appelée Menologium rusticum Colotianum cette indication dépourvue d'ambiguïté : " mois de novembre, semailles du froment et de l'orge - mensis november : sementes triticariae et hordiar(iae) ${ }^{61}$." Virgile a mis en forme de doctrine la pratique de ses compatriotes : "Si c'est pour le froment et les robustes gerbes de l'épeautre que vous labourez la terre, si vous ne souhaitez qu'une riche moisson d'épis, attendez pour ensemencer vos sillons que les Atlantides se couchent à l'aurore :

At si triticeam in messem robustaque farra

Exercebis humum solisque instabis aristis,

Ante tibi Eoae Atlantides abscondantur

Debita quam sulcis committas semina ${ }^{62}$."

Les filles d'Atlas se confondent en ces vers des Géorgiques avec les Pléiades, dont la constellation, associée à celle des Hyades, leurs célestes voisines et leurs sœurs de la mythologie, est appelée en latin Vergiliae. Par une tradition constante, les anciens espa-

\footnotetext{
60 I, 373-376.

61 C.I.L., $\mathrm{I}^{2}$, p. $280=$ VI, $2305=$ DesSAU, I.L.S., 8745. Le nom de Colotianum est dérivé de celui de l'humaniste du XVI ${ }^{\mathrm{e}}$ siècle qui acquit le document.

62 VIRG, Georg., I, 219-222.
} 
çaient le coucher matinal de ces étoiles - matutinus occasus Vergiliarum - du 26 octobre, où il commence selon Columelle ${ }^{63}$, au 11 novembre, où il s'achève selon Pline ${ }^{64}$.

C'est le coucher des Vergiliae qui indique au semeur que le moment est venu de remplir sa tâche : il commencera par le blé quand, au matin, se coucheront les Vergiliae : incipiemus a sementibus frumenti Vergiliarum occasu matutino ${ }^{65}$. Dans ces conditions, on doit bien penser que c'est aussi en novembre que les paysans de Faleria célébraient la fête de leurs semailles.

D'après Rutilius, ils l'associaient au culte d'Osiris et s'abandonnaient à la joie d'avoir enfin " retrouvé " vivant le dieu par qui germent les semences et grandissent les futures moissons. Or, précisément les calendriers rustiques - menologia rustica -, à l'un desquels - le Colotianum - j'ai fait tout à l'heure allusion, assignent au mois de novembre la retrouvaille d'Osiris : mensis november, (h)euresis ${ }^{66}$. Singulière puissance d'attraction des religions de l'Egypte hellénistique ! Elles se sont répandues jusque chez les paysans latins et ils ont voué à ces rites et à ces mystères orientaux une incroyable fidélité. Les menologia rustica où s'inscrit, sous son nom grec latinisé, l' heuresis osirienne ont été gravés au $\mathrm{I}^{\mathrm{er}}$ siècle de notre ère. $\mathrm{Au} \mathrm{V} \mathrm{V}^{\mathrm{e}}$, le texte de Rutilius est là pour attester qu'elle se célébrait toujours, dans les moindres bourgades d'Italie, avec la même fervente allégresse. Les rites originaux de cette fête nous sont décrits au chapitre $39 \mathrm{du}$ traité de Plutarque de Iside et Osiride. Osiris a disparu au mois d'Athyr, dans la période où les vents étésiens ne soufflent plus, où le Nil, s'enfonçant dans la terre, laisse le sol à nu, où, l'obscurité grandissant, la lumière paraît comme vaincue. Alors les prêtres, pour figurer le deuil d'Isis, recouvrent une vache dorée d'une draperie de lin noir et ils l'exposent, durant quatre jours de suite, à partir du 17 du mois ; le 19 Athyr, quand la nuit est venue, la population descend vers la mer. Les stolistes et les prêtres apportent une ciste sacrée qui contient une petite boite en or, dans laquelle ils versent de l'eau douce. De l'assistance, à cet instant, s'élève une clameur et tous crient qu'Osiris vient d'être retrouvé ${ }^{67}$.

De ce passage, il ressort que les fêtes de l'heuresis s'étendent sur quatre jours. Osiris est censé perdu pendant les trois premiers, le 17, le 18 et le 19 Athyr ; et il est censé retrouvé au soir du 19 Athyr. Dans ces conditions, il est nécessaire, pour compléter le quatriduum dont parle Plutarque, que le lendemain, c'est-à-dire le 20 Athyr, ait été consacré aux réjouissances qu'a provoquées la résurrection divine. En outre, il résulte d'un autre passage du même traité De Iside et Osiride que le mois d'Athyr est, pour Plutarque, un mois fixe de l'année stabilisée d'Alexandrie ; le mois des

63 Columelle, II, 8, 2 : absconduntur altero et trigesimo die post autumnale aequinoctium.

64 PLINE, N.H., XVIII, 113 : iii id(us) novembres Vergiliae occidunt.

65 PLINE, N.H., XVIII, 223.

66 Cf. C.I.L., I ${ }^{2}$, p. $280=$ VI, $2305=$ DeSSAU, I.L.S., 8745.

67 Je modifie à peine la traduction du chapitre par Mario Meunier, Paris, 1924, p. 130. 
semailles, appelé Pyanepsion par les Grecs ${ }^{68}$. Pour Plutarque, par conséquent, Athyr, comme Pyanepsion, commence en un jour qui correspond à la mi-octobre pour finir à la mi-novembre du calendrier Julien ${ }^{69}$. D'où il suit que, selon Plutarque, les fêtes osiriennes duraient du 31 octobre au 3 novembre : les Égyptiens pleuraient la disparition d'Osiris le 31 octobre, le ler et le 2 novembre. Ils retrouvaient leur dieu à la nuit tombante du 2 novembre et ils passaient en festivités la journée du 3 novembre. En Égypte, d'après Plutarque et de son temps, la fête d'allégresse tombait le 3 novembre. Elle s'est à coup sûr maintenue telle quelle aux époques suivantes. Elle a émigré en Italie. Mais, en changeant de théâtre et de calendrier, elle a conservé sa place immuable dans le mois des semailles et des pluies méditerranéennes. Dans son traité De mensibus, le Byzantin Laurentius Lydus notera encore : "Le 2 et le 3 novembre, dans le temple

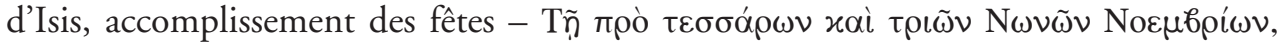

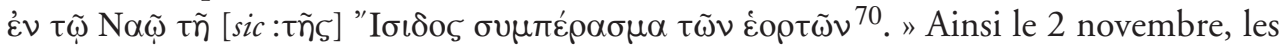
dévots continuaient, au Bas-Empire, de pleurer, dans la perte d'Osiris, la mort de la nature entière ; le 3, ils continuaient de saluer la trouvaille du dieu ressuscité comme le gage du renouveau du monde; et ils se livraient, en son honneur, à de tels transports que, de la religion d'Attis, où des hilaria se célébraient depuis le règne de l'empereur Claude ${ }^{71}$, la joie s'en transmit à l'heuresis osirienne. Au IV siècle de notre ère, Philocalus assigne, sur son calendrier, le troisième jour des nones de novembre, soit le 3 novembre, aux hilaria ${ }^{72}$, avec quoi elle se confond alors et dont l'écho se répercute dans le vers de Rutilius Namatianus : "Alors il se trouva que les paysans hilares étaient répandus dans leurs carrefours rustiques :

Et tum forte hilares per compila rustica pagi ${ }^{73}$."

Enfin, nous tenons ici le point immobile où suspendre le développement du voyage raconté par le poète. À Faleria, Rutilius est survenu au beau milieu des hilaria de l'heuresis. C'est-à-dire qu'il y débarqua un 3 novembre ; et comme cette escale termine le quatrième jour de sa traversée, il a laissé le Portus Ostiensis à l'aube du 31 octobre, et il est sorti de Rome quinze jours auparavant, un 16 octobre, par conséquent. Cette conclusion effarouchera peut-être par sa rigueur, mais d'autres détails du poème, que les savants de la chronologie courante ont tant de peine à expliquer, vont la confirmer de leurs convergences spontanées.

68 Plut., De $I s$. et Os., LXIX.

69 Contra, mais à tort, d'après Mommsen, Wissowa, Religion u. Kultus der Römer ${ }^{2}$, p. 353-354.

70 Lydus, De mens., IV, 148, p. 166, Wuensch. Lydus est postérieur d'un siècle à Rutilius : la persistance, à son époque, de l'heuresis aux quatre et trois des nones de novembre (traduction littérale de sa chronologie) n'en est que plus frappante.

71 Cf. mes Aspects mystiques, notamment p. 53.

72 C.I.L., $\mathrm{I}^{2}$, p. 260.

73 I, 373 ; cf. supra, p. 250 [ici : p. 251]. 
Examinons les plus instructifs.

$1^{\circ}$ Pendant son attente dans le Portus Ostiensis, Rutilius, non sans surprise, a perçu dans le lointain l'écho des jeux du Cirque. Les acclamations redoublées lui prouvent que l'affluence est au comble : "Plus d'une fois, à mes oreilles étonnées, retentissent les jeux du Cirque : ces flambées d'enthousiasme m'annoncent que les gradins sont pleins :

Saepius attonitae resonant Circensibus aures,

Nuntiat accensus plena theatra favor ${ }^{74}$."

Or, en septembre, les calendriers du Bas-Empire n'indiquent de ludi circenses qu'avant l'équinoxe. Philocalus, en 354, en note encore le 20, le 21 et le 22 du mois. Polemius Silvius, en 448, n'en note plus que le 20 septembre. Après quoi, ni l'un ni l'autre n'en mentionnent plus avant le 20 octobre. C'est pour se mettre en règle avec ces témoignages que Vessereau et Vollmer ont refoulé l'équinoxe sur le 21 ou le 22 septembre, à la moderne. Mais si, de la sorte, ils se sont entendus avec Philocalus, ils restent en délicatesse avec Polemius Silvius ; et ils contreviennent de toute façon, à l'indication formelle des documents anciens unanimes à situer au 24 septembre l'équinoxe d'automne. Respectueux de leur concordance, Scaliger avait attendu jusqu'au 24 septembre pour mettre Rutilius en route. Mais comment le poète aurait-il pu entendre, au Portus Ostiensis, s'il n'y est arrivé que le 24, le fracas de spectacles qui remontent au 20, au 21 ou même au 22 septembre ? À partir du 10 octobre, au contraire, nous n'avons que l'embarras du choix entre les ludi circenses inscrits au calendrier : ludi Alemannici circenses le 10 ; ludi Augustales circenses le 11; ludi Iovis circenses le 18 ; ludi Solis circenses le 22 ; ludi adventus Divi circenses le 29 octobre. À parcourir, dans les appendices de Wissowa ${ }^{75}$, cette énumération on conçoit aisément que Rutilius, séjournant au Portus Ostiensis du 16 au 31 octobre, y ait eu, à diverses reprises, l'occasion de tendre l'oreille aux rumeurs qui, pendant cette période, remplissaient le Cirque romain.

$2^{\circ}$ Rutilius aurait voulu quitter tout de suite le Portus Ostiensis. Mais, bloqué par le mauvais temps, il dut y attendre la première embellie ramenée par la nouvelle lune :

\section{Dum melior lunae fideret aura lunae ${ }^{76}$.}

Les érudits ont naturellement cherché au ciel de l'an 416 cette lune bienfaisante. Corsini l'a découverte le 7 octobre ; Zumpt le 8, Scaliger le 9 ; et Vessereau n'a pas essayé de les départager ${ }^{77}$. Mais puisque, nous l'avons vu, Rutilius n'a quitté Rome

74 I, 201-202.

75 Wissowa, op. cit., p. 589.

76 I, 206.

77 Vessereau, op. cit., p. 256. C'est Corsini qui, d'ailleurs, avait raison d'après les tables de Guinness citées ci-après. 
qu'un an plus tard, les recherches de Scaliger, Corsini et Zumpt sont également stériles, et ruineuses les conclusions qu'après eux Vessereau en a déduites. Leurs calculs sont à recommencer sur la seule base qui nous soit dorénavant consentie : celle de l'an 417 . Or, en 417 , dont l'épacte est définie par le chiffre $18^{78}$, la nouvelle lune s'est levée le 27-28 septembre, trop tôt pour nous permettre de retarder au 8 ou 9 octobre l'embarquement qu'elle a favorisé, et le 26-27 octobre $^{79}$ soit juste à point pour apaiser les ilôts auxquels, suivant mon propre raisonnement, Rutilius s'est enfin confié, à l'aube du 31 octobre. Ainsi les indices ${ }^{80}$ que la chronologie régnante est accoutumée d'invoquer se retournent contre elle et fortifient ma conviction ; et, décidément assuré que Rutilius a relâché à Faleria le 3 novembre 417, je restitue son emploi du temps ainsi qu'il suit :

1) Départ de Rome et arrivée au Portus Ostiensis = 16 octobre 417 ;

2) Départ du Portus Ostiensis = 31 octobre 417, à l'aube ;

3) Escale de Centumcellae $=31$ octobre 417 (après-midi) ;

4) Escale du Portus Herculis $=\mathrm{I}^{\mathrm{er}}$ novembre 417 ;

5) Escale de l'embouchure de l'Umbro = 2 novembre 417 ;

6) Escale de Faleria $=3$ novembre 417 ;

7) Escale de Populonia $=4$ novembre 417 ;

8) Escale de Vada $=5$ novembre 417.

À partir de là, les voies de Rutilius sont quelque peu brouillées. Tandis que jusqu'alors il avait franchi autant d'étapes qu'il avait compté de jours, il fut désormais contraint de compter avec l'état de la mer : le rythme, auparavant uniforme, de ses navigations, se brisa contre le mauvais temps et la houle.

Pour demeurer conséquent avec son programme, il aurait dû lever l'ancre à Vada, le 6 novembre au matin. Il n'en put rien faire : du Nord-Ouest, s'était levé un violent Corus qui lui interdit de reprendre la mer aussitôt : " Là, je dus m'arrêter devant un Corus impétueux, comme il l'est lorsqu'il brise les halliers bourbeux des forêts :

Illis me rapidus consistere Corus adegit,

Qualis silvarum frangere lustra solet ${ }^{81}$."

78 Cf. Giry, Manuel de diplomatique, p. 184.

79 Cf. Grassan Guinness, Creation centred in Christ, Londres, 1896, II, p. 340.

80 Je n'insiste pas sur la mention des Chelae (I, 156), ou "pinces du Scorpion ", car si le signe du Scorpion n'est invocable qu'à partir du 19 octobre, il est possible qu'elles remplacent ici la Balance, dont le signe intervient un mois plus tôt. Le vers I, 187 - occidua Plias prouve que les Vergiliae inclinent vers leur couchant, lequel commence le 26 octobre (cf. supra, p. 251 [ici : p. 251 aussi]).

81 I, 463. 
Hébergé, pour la nuit, dans la villa que possédait à Vada le Decius Albinus qui, en 414, lui avait succédé dans la préfecture de la Ville, il y éprouva bientôt la consolation d'y être rejoint le lendemain, 6 novembre, par l'un de ses amis, Victorinus, de Toulouse $^{82}$. «Pendant que je l'embrassais, nous confie cet homme sensible, j'oubliais les vents contraires : il me semblait jouir déjà d'un avant-goût de ma patrie :

\section{Hune ego complexus ventorum adversa fefelli,}

Dum videor patriae iam mihi parte frui ${ }^{83}$."

La rencontre des deux " compatriotes " ne parait pas, du reste, s’être prolongée, et Rutilius l'abrège en ce vers d'une jolie préciosité : «L'amère tempête nous valut un doux retard :

\section{Tempestas dulcem fecit amara moram ${ }^{84}$. "}

Après avoir serré le Toulousain Victorinus sur son cœur, le poète renoue le fil de son odyssée et remonte à son bord, aux premiers feux du jour : «L'Aurore aux doigts de roses avait poussé son attelage dans la lumière du ciel : la brise du rivage nous persuade de tendre nos antennes :

\section{Lutea protulerat sudos Aurora ingales:}

Antemnas tendi litoris aura iubet ${ }^{85}$."

Tout lecteur non prévenu partagera l'impression de Vessereau que la halte de Vada n'a pas dépassé un jour et que, dans la matinée du 7 novembre, Rutilius a vogué sur la Tyrrhénienne. Dans l'après-midi, il atterrissait, à proximité du port de Pise, dans la villa de Triturrita, préparée pour l'accueillir :

Inde Triturritam petimus ${ }^{86} \ldots$

S’il n'eût écouté que la sagesse, il eût, sans désemparer, poursuivi sa route, car le vent du Sud-Est, l'Eurus, lui promettait, sous un ciel limpide, une navigation favorable :

Tempora navigiis clarus reparaverat Eurus ${ }^{87}$.

Mais, chez Rutilius, l'envie l'emporta de visiter Pise, où s'érigeait une statue de son père Lachanius, et d'y revoir le Trévire Protadius qui s'y était porté à sa rencontre. Il remise ses embarcations dans un abri sûr et se fait conduire, à pied, jusqu'à la ville voisine :

82 I, 509-510. Ces vers sont, du reste, à verser au dossier des origines toulousaines de Rutilius.

83 I, 492.

84 I, 511.

85 I, 559-560.

86 I, 527.

87 I, 541. 


\section{Puppibus ergo meis fida in statione locatis, Ipse vehor Pisas qua solet ire pedes ${ }^{88}$,}

Négligeant l'hospitalité qu'on lui offrait à Triturrita, il s'est donc rendu à Pise, où il a couché le 7 novembre au soir. La journée du 8 s'est écoulée à Pise où, sûrement, il avait donné rendez-vous à Protadius, dont le domaine, qu'il ne cite que pour mémoire et sans l'avoir visité, était sis en Ombrie ${ }^{89}$. Après quoi, sans transition, il nous conte son retour à Triturrita, lequel s'est effectué, de toute évidence, le soir même du 8 novembre. Seulement, dans l'intervalle, le vent avait tourné. L'Africus, le Libeccio d'aujourd'hui, s'était déchaîné du Sud-Ouest, forçant Rutilius à différer son rembarquement. Logique avec lui-même, Vessereau, qui a situé au 15 octobre le séjour à Pise $^{90}$ et qui se voit obligé, par une précision astronomique sur laquelle nous devrons tout à l'heure revenir, à refouler sur la fin de la première quinzaine de novembre le départ de Triturrita, conclut que la halte en cette villa pisane a duré tout un mois ${ }^{91}$. Mais cette conclusion est démentie par les vraisemblances et par le texte du poème. Si le séjour près du port de Pise s'était prolongé deux fois plus que le séjour au Portus Ostiensis $^{92}$, s'il avait absorbé quatre fois plus de temps que tout le reste du voyage, Rutilius, assurément, n'aurait pu oublier des délais aussi insolites et nous en eût dit un mot. Au contraire, il traite ce second retard à peu près comme, naguère, celui de Vada. Ces deux arrêts forcés ont dû être de longueur sensiblement analogue. Celui de Vada, qui tient en vingt vers ${ }^{93}$, avait duré trente-six heures et fut coupé par la visite de Victorinus. Celui de Triturrita, raconté en trente et un vers, est rempli par la narration d'une chasse au sanglier ${ }^{94}$, et par la description de l'ouragan ${ }^{95}$. Ce fut, apparemment, l'affaire de deux jours pleins, tout au plus.

Aussi bien une tempête d'Africus sévissant un mois d'affilée ne s'est-elle jamais vue. En la laissant s'apaiser au bout de trois jours, on rentre, au contraire, dans les conditions habituelles où se produisent les bourrasques du Libeccio. Si celle dont a pâti Rutilius s'est déclarée, comme il le dit, pendant qu'il se promenait dans Pise, c'est-àdire le 8 novembre, elle a dû, normalement, tomber au soir du 10 novembre ; et c'est, partant, le 11 novembre, au matin, que s'effectua la dernière traversée dont nous parle Rutilius, celle qui l'a amené du port de Pise dans celui de Luna : "Glissant à vive allure sur les flots, nous arrivons à des murailles éclatantes de blancheur : elles tirent leur nom de la sœur du soleil dont elle emprunte les rayons :

88 I, 559-560.

89 I, 151 : Substituit patriis mediocres Umbria sedes.

90 Vessereau, op. cit., p. 267.

91 Ibid., p. 268.

92 Cf. supra, p. 256 [ici : p. 254].

93 I, 491-510.

94 I, 615-630.

95 I, 630-644. 


\section{Advehimur celeri candentia moenia lapsu}

Nominis est auctor sole corusca soror ${ }^{96}$."

Et, sur cette vision de rêve éblouissante, Rutilius nous abandonne.

En résumé, s'il nous est interdit de jalonner la fin de son itinéraire avec la même précision que le commencement, il parâtra plausible d'en dater les derniers incidents ainsi qu'il suit :

- Bourrasque de Corus à Vada $=6$ novembre 417 ;

- Départ de Vada ; arrivée à Triturrita de Pise $=7$ novembre 417 ;

- Visite de Pise, rencontre avec Protadius, commencement de la bourrasque d'Africus $=8$ novembre 417 ;

- Retour à Triturrita $=$ soir du 8 novembre 417 ;

- Chasse au sanglier $=9$ novembre 417 ;

- Continuation et fin de la bourrasque d'Africus $=9$ et 10 novembre 417;

- Départ de Triturrita et arrivée à Luna $=11$ novembre 417.

À quoi bon, dira-t-on peut-être, à quoi bon épiloguer sur de pareils détails ? Ces données minuscules et contingentes ne seront jamais que conjecturales et, au surplus, en quoi pourraient-elles intéresser la personnalité du poète et l'intelligence de son ouvrage?

Tel est le jugement que l'on formule d'ordinaire et contre lequel il me faut protester.

Je suis, en effet, persuadé que, dégagée d'hypothèses, qui se sont vérifiées l'une après l'autre, la vérité qu'elles renferment vient éclairer l'énigmatique figure de l'auteur et rénover notre interprétation de son poème.

Dans le tableau de la tempête qu'a subie Rutilius à Pise avant de cingler vers Luna, le poète a inséré une digression savante : "Déjà, se couchent, dans le matin, les Hyades humides ; déjà, sous des pluies d'hiver, disparaît le Lièvre, astre faible par ses rayons et assez fort pour soulever les flots et empêcher les marins de s'éloigner de la terre inondée ; c'est qu'il est le voisin immédiat du tempétueux OrIon et qu'il fuit, proie mouillée, devant le CHIEN brûlant :

Iam matutinis Hyades occasibus udae.

Iam latet hiberno conditus imbre Lepus,

Exiguum radiis, sed magnis fluctibus astrum,

Quo madidum nullus navita linquit humum;

Aestiferumque Canem roscida praeda fugit ${ }^{97}$. "

96 II, 63-64.

97 I, 633-638. 
Il y a là tout un docte résumé d'astronomie météorologique. Mais dans son empressement à nous étaler sa science, Rutilius a rapproché des dates qui ne concordent pas. Que l'on consulte plutôt les tables de Boll ${ }^{98}$ : le coucher du Chien a lieu le 7 décembre ; celui du Lièvre le 21 novembre. Il est clair que cette nomenclature a été déclenchée par l'évocation des Hyades auxquelles ces diverses constellations succèdent sur la carte du ciel et dans leurs mouvements apparents. Le coucher matinal des Hyades importe seul et c'est là l'indice chronologique auquel nous devons nous attacher exclusivement ${ }^{99}$. Filles d'Atlas, comme les Pléiades, contiguës aux Pléiades sur la voûte étoilée, les Hyades obéissent au commun destin des Atlantides chantées par Virgile ${ }^{100}$ et que les auteurs latins désignent communément sous le vocable de Vergiliae. Comment d'ailleurs distinguerait-on leurs révolutions et leurs vertus ? "Semez au temps des Hyades ", conseillait déjà Hésiode ${ }^{101}$. Au temps du coucher des Pléiades, "semez " répètent Virgile ${ }^{102}$ et Columelle ${ }^{103}$. Sur le ciel d'Athènes, les Pléiades disparaissent au matin du 8 novembre; sur celui de Rome, les Hyades s'évanouissent le 10 et les Pléiades le 13 novembre ${ }^{104}$. À l'ordinaire, les Latins fixent au 11 novembre la fin du coucher des Vergiliae : tertium idus (novembres) Vergiliae occidunt ${ }^{105}$. Ce calcul conventionnel coïncide trop exactement avec la date que, par une autre voie, nous avons réussi à assigner à la dernière escale de Rutilius, pour ne point la confirmer avec éclat : le poète a sûrement accompli la dernière de ses étapes, celle de Pise à Luna, le 11 novembre.

Or, pour toute l'antiquité et surtout au Bas-Empire romain, le 11 novembre a marqué l'arrêt obligatoire de la circulation maritime normale. Pour Euripide, déjà, le coucher des Hyades en donnait le signal aux matelots de son temps ${ }^{106}$. Huit siècles après lui, à l'époque même où vécut Rutilius, Végèce atteste que telle est toujours la réalité. "Le coucher hivernal des Vergiliae, déclare-t-il sans ambages, bouleverse la navigation : en conséquence de quoi, depuis le 11 novembre jusqu'au 10 mars, les mers sont fermées : navigia conturbat Vergiliarum biemalis occasus : eo die igitur, tertio idus novembres usque in diem sextum idus martias maria clauduntur ${ }^{107}$. "

Cette fermeture de la mer, coïncidant avec les derniers vers de Rutilius, ne marque pas seulement la fin préméditée du poème. Du même coup, elle va nous permettre de

98 Boll, s. v ${ }^{\circ}$, Fixsterne, P.W., VI, c. 2429-2430.

99 C'est ce qu'a fait Vessereau, à bon droit, mais à tort pour l'année 416 ; cf. supra, p. 256 [ici : p. 254].

100 Cf. supra, p. 250 [ici : p. 251].

101 Hésiode, Trav., 615. (Les Hyades sont nommées avec les Pléiades.)

102 VIRG., Georg., I, p. 219 et suiv.

103 Columelle, II, 8, 2 ; cf. supra, p. 251 [ici : p. 252].

104 Boll, op. cit., loc. cit. Ses tables nous reportent à 430 av.J.-C. pour Athènes ; à 45 av. J.-C. pour Rome.

105 Pline, N.H., XVIII, 213.

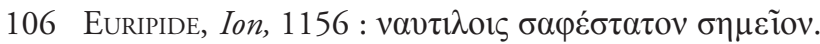

107 VÉGÈCE, IV, 39. 
résoudre tous les problèmes que soulève la composition de l'ouvrage dont, en commençant, nous avons cerné les contours.

D'abord, grâce à ce finale visiblement concerté, il nous apparait que le poète, dans la paix retrouvée en ses domaines de Gaule, a versifié de loisir le récit de la traversée par laquelle il a inauguré son retour en sa patrie. Mais, en même temps, il est certain qu'il chercha à lui communiquer, en nombre de ses distiques, le ton d'un carnet de bord, à lui imprimer l'allure d'une improvisation poursuivie, au jour le jour, d'escale en escale. Avec sa finesse habituelle, le regretté Pierre de Labriolle nous a prémuni contre les artifices de ce trompe-l'œil ${ }^{108}$. Toutefois, nous ne devons nullement retenir la qualification de sottise décochée par Vollmer contre Vessereau ${ }^{109}$, parce que celui-ci s'est laissé prendre à l'illusion que le poète avait cherché à créer et par laquelle son talent parvint, ici et là, à tromper ses lecteurs ${ }^{110}$.

En même temps, le hasard est éliminé d'une mutilation accidentelle qui nous aurait dérobé la fin du poème. Le début embarrassé du livre $n$ nous prépare à la coïncidence des derniers distiques avec l'escale de Luna qui ne pouvait être suivie d'aucune autre ; et les hésitations qu'il exprime montrent, que Rutilius savait d'avance qu'il ne pourrait conduire son livre II aussi loin que le premier : « Nous répartissons entre deux petits ouvrages les rougeurs tremblantes d'un travail qu'il eût mieux valu contenir en un seul :

\section{Partimur trepidum per opuscula bina ruborem}

Quem satius fuerat sustinuisse semel ${ }^{111}$."

Pour comprendre la confusion dont rougit Rutilius et les perplexités d'édition qu'il avoue, il n'est pas besoin de supposer que, soit les ultimes feuillets du manuscrit que nous acquit le larcin de Bonneval Pacha, soit les derniers feuillets de l'archétype, furent jadis arrachés et sont aujourd'hui perdus à jamais. À plus forte raison, est-il superflu de s'approprier le petit roman que Vollmer ridiculise à bon droit ${ }^{112}$ et suivant lequel Rutilius aurait péri en cours de route, sorte de Camoëns malheureux dont on n'aurait sauvé du naufrage que des Lusiades inachevées. Le Gaulois, né malin, a apposé sur son œuvre le point final où celui-ci était nécessaire ; et le secret de ses intentions se découvre dès que l'on observe que son récit s'arrête net le jour où la fermeture annuellement obligatoire des mers a mis fin à son périple.

Ce serait se refuser à la vision concrète des faits que de croire que les deux petits ouvrages - bina opuscula - dont parle le poète ce prouveraient l'existence de deux iusta

108 Labriolle, R.E.L., 1928, p. 31 ; cf. supra, p. 236 [ici : p. 243].

109 Vollmer, P.W., I $\mathrm{I}^{\text {A }}$ c. 1251

110 Vessereau, op. cit., p. 254 : Le poème dut être composé au jour le jour, on pourrait presque dire heure par heure.

111 II, 9-10.

112 Vollmer, op. cit., loc. cit. 
volumina sensiblement égaux ${ }^{113}$. En effet, regardons la carte et reportons sur elle les distances que Rutilius a franchies en bateau :
Du Portus (Porto) à Centumcellae (Cività Vecchia) $=80 \mathrm{~km}$
De Centumcellae à Portus Herculis
$=70 \mathrm{~km}$
De Portus Herculis à l'Umbro
$=30 \mathrm{~km}$
De l'Umbro à Faleria ${ }^{114}$
$=70 \mathrm{~km}$
De Faleria à Populonia
$=25 \mathrm{~km}$
De Populonia à Vada
$=40 \mathrm{~km}$
De Vada à Pise
$=80 \mathrm{~km}$
De Pise à Luna
$=60 \mathrm{~km}$

Le poète a donc mis 26 jours pour couvrir $425 \mathrm{~km}$. Il ne se pressait pas ; et il n'a jamais demandé aux petites embarcations, sur lesquelles lui et sa suite étaient montés ${ }^{115}$ l'effort que de plus gros bateaux auraient fourni en portant aisément à plus de cent kilomètres par journée la moyenne de leurs navigations ${ }^{116}$. Mesurons maintenant la route terrestre que Rutilius pour gagner Toulouse a dû suivre, avec une lenteur accrue du simple au double ou, plutôt, du simple au triple ${ }^{117}$ ! Comme elle s'étire sur près de 800 kilomètres de longueur, ce n'est pas un second livre, égal au premier, mais au moins deux livres de plus qu'il aurait écrits, s'il avait multiplié avec la même facilité le nombre de ses étapes en terre gauloise et détaillé chacune d'elles avec la même complaisance.

Il est vrai qu'à l'appui d'une opinion que ruinent ces calculs élémentaires, $M$. Préchac a partagé le sentiment émis par Pierre de Labriolle, suivant lequel, au vers 62 du livre II, Rutilius s'apprêtait "à prendre comme un nouvel élan ${ }^{118}$ ". Quant à moi, j'en retire l'impression contraire, et je crois que le poète y a marqué les limites qu'il avait posées d'avance à ses narrations. Quand, pour s'excuser de la double digression

113 Comme le veut PrÉCHAC, op. cit., p. XXI-XXII.

114 Précisément Falesia Portus, cf. Huelsen, P.W., VI, c. 1072 ; cf. supra, p. 250 [ici : p. 251].

115 « Nous avançons le long de rivages tout proches, sur de petites barques capables de trouver un refuge dans les nombreuses échancrures de la côte. "

116 Cf. mon Maroc antique, p. 121, n. 6.

117 M. Pflaum, dont l'érudition est inépuisable, a bien voulu grouper à mon intention aussi bien les textes utilisés dans le livre classique de Riepl que dans son propre mémoire sur le Cursus publicus (ou Poste impériale).

Or, de cette confrontation, il ressort : $1^{\circ}$ qu'on ne saurait envisager ici les vitesses record, signalées comme des exceptions, qui totalisent, à pied comme à cheval, 80 à $90 \mathrm{~km}$ par jour ; $2^{\circ}$ qu'on doit prendre pour base de calcul la vitesse de 20 milles $=30 \mathrm{~km}$ par jour, indiquée aux justiciables qui doivent répondre à une citation du préteur (Dig., II, 11, 1).

Dans ces conditions, si Rutilius avait marché sans arrêt, il eût mis une trentaine de jours pour gagner Toulouse, mais il a dû se ménager en chemin plus d'une confortable étape ; et l'on sera très près de la vérité en admettant que son retour terrestre dans sa patrie gauloise lui a demandé trois mois (90 jours au minimum).

118 Labriolle, op. cit., loc. cit., p. 31 ; et Préchac, op. cit., p. XXII. 
où il vient de se laisser entraîner - son éloge de la structure de la péninsule italienne ${ }^{119}$ et son invective contre Stilichon ${ }^{120}$-, Rutilius s'écrie : «Et maintenant, reprenons la route dont notre poème s'est proposé la description :

Carmine propositum iam repetamus iter ${ }^{121} "$,

il répète simplement, pour finir, le dessein qu'il avait énoncé en commençant, celui de raconter en vers la navigation, qu'il avait préférée au voyage par terre : "Si tard que ce soit, nous nous résignons au voyage par mer que nous avons choisi, parce que, sur les chemins terrestres, les plaines sont inondées par les fleuves et les hauteurs hérissées de rochers, etc. :

... Et serum vix toleramus iter,

Electum pelagus quoniam terrena viarum

Plana madent fluviis, cautibus alta rigent ${ }^{122}$."

Le voyage par mer - iter marinum, ou maritimum - voilà quel fut le sujet de Rutilius, et le poète l'a traité intégralement, sans vouloir en aborder un autre : "Mon rouleau n'était pas bien long, ne comprenant que peu de tours. Il aurait pu, naturellement, s'allonger encore. Mais il a craint l'ennui d'un interminable travail et n'a pas voulu effrayer le lecteur d'un débit qui ne tarirait plus :

Nondum longus erat nec multa volumina passus

Iure suo poterat longior esse liber.

Taedia continuo timuit cessura labori,

Sumere ne lector inge paveret opus ${ }^{123}$."

Cette fois la cause est jugée, et par Rutilius lui-même ; et il va s'ensuivre qu'au moins, par son incipit, le manuscrit le plus récent, le Romanus, est supérieur au Vindobonensis. Le copiste de celui-ci jugeait, à tort, que l'ouvrage, qu'il avait transcrit, n'était que fragmentaire ; ex fragmentis Rutilii ${ }^{124}$. Le copiste de celui-là, mieux informé $^{125}$, savait que, dédié à Rufius Volusianus, l'ouvrage était complet ; et il l'a désigné par un substantif : itinerarium, qui ne saurait se suffire à lui-même, mais sous lequel un épigraphiste n'éprouvera guère de difficulté à déceler le texte qu'il a estropié. Dans les inscriptions, les adjectifs marinus, maritimus s'abrègent en mar ${ }^{126}$; le

\footnotetext{
119 II, 15-39.

120 II, 40-61.

121 II, 62.

122 I, 36-38.

123 II, $1-4$.

124 Cf. PRÉCHAC, op. cit., p. 2.

125 À noter que les Romanus présente « en un petit nombre de passages des leçons plus rapprochées de la tradition authentique " (PréCHAC, op. cit., p. xxv).

126 Cf. Cagnat, Manuel...4 , p. 444.
} 
groupe est facile à confondre avec inar : et l'on conçoit aisément que l'itinerarium du Romanus soit sorti, par mauvaise lecture, des deux mots que portait son modèle : iter mar(itimum). C'est là, sans doute, la transcription originale et véridique du titre que Rutilius avait choisi pour son poème, parce que celui-ci correspondait exactement au contenu de l'ouvrage et au dessein que le poète avait conçu et conduit à son terme. 